\title{
Erratum: Systematic investigation of low-lying dipole modes using the canonical-basis time-dependent Hartree-Fock-Bogoliubov theory [Phys. Rev. C 90, 024303 (2014)]
}

\author{
Shuichiro Ebata, Takashi Nakatsukasa, and Tsunenori Inakura
}

(Received 20 August 2015; published 27 October 2015)

DOI: 10.1103/PhysRevC.92.049902

PACS number(s): 21.10.Pc, 21.60.Jz, 24.30.Gd, 25.20.-x, 99.10.Cd

We have found that the ground-state properties of ${ }^{100} \mathrm{Kr}$ and ${ }^{76} \mathrm{Sr}$ in Tables II and III of the original paper are not of the ground state but of the local minima. The correct values for the ground state are given in the following Tables II and III. In the original paper, the electric dipole strength functions of ${ }^{100} \mathrm{Kr}$ and ${ }^{76} \mathrm{Sr}$ were calculated on each of the local minima. Accordingly, Fig. 13 should be replaced by the following. However, we have confirmed that all the arguments given in the original paper are still valid.

TABLE II. Ground-state properties of ${ }^{100} \mathrm{Kr}$ obtained by the $\mathrm{HF}+\mathrm{BCS}$ with $\mathrm{SkM}^{*}$; quadrupole deformation parameters $\left(\beta_{2}, \gamma\right)$, pairing gaps for neutrons and protons $\left(\Delta_{n}, \Delta_{p}\right)(\mathrm{MeV})$, and chemical potentials for neutrons and protons $\left(\lambda_{n}, \lambda_{p}\right)(\mathrm{MeV})$. The root-mean-square radii of neutrons and protons $\left(r_{n}, r_{p}\right)(\mathrm{fm})$ are also shown.

\begin{tabular}{cccccccrr}
\hline \hline & $\beta_{2}$ & $\gamma$ & $r_{n}$ & $r_{p}$ & $\Delta_{n}$ & $\Delta_{p}$ & $-\lambda_{n}$ & $-\lambda_{p}$ \\
\hline${ }^{100} \mathrm{Kr}$ & 0.36 & $0^{\circ}$ & 4.69 & 4.36 & 1.35 & 1.43 & 3.98 \\
\hline \hline
\end{tabular}

TABLE III. The same as Table II but for ${ }^{76} \mathrm{Sr}$.

\begin{tabular}{ccccccccc}
\hline \hline & $\beta_{2}$ & $\gamma$ & $r_{n}$ & $r_{p}$ & $\Delta_{n}$ & $\Delta_{p}$ & $-\lambda_{n}$ & $-\lambda_{p}$ \\
\hline${ }^{76} \mathrm{Sr}$ & 0.00 & & 4.04 & 4.12 & 2.27 & 2.28 & 13.53 \\
\hline \hline
\end{tabular}

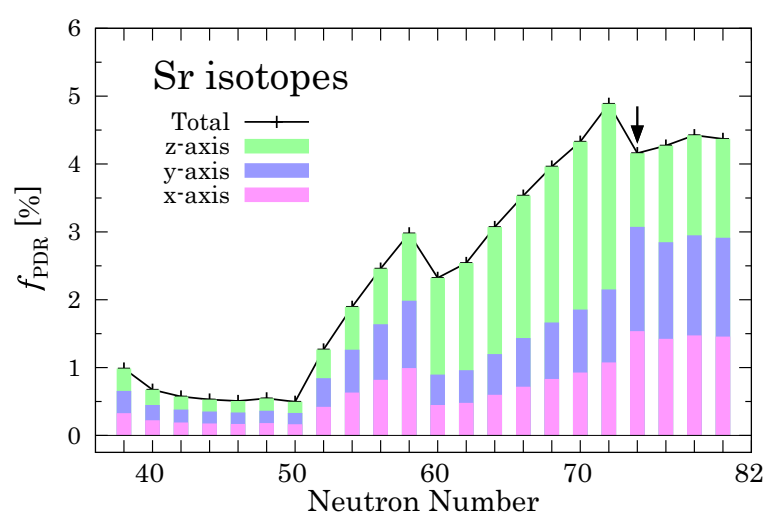

FIG. 13. (Color online) The pygmy dipole resonance strength in Sr isotopes decompose into $x, y$, and $z$ components and are denoted by red, blue, and green bars. In spherical nuclei, the three components are equal to each other. In this figure, we include isotopes with the neutron chemical potentials smaller than $2 \mathrm{MeV}(N>82)$. The arrows indicate the oblate-shaped nuclei. 